\title{
The Simulation and Study of the Operating System Efficiency Improvement of a Container Terminal
}

\author{
Di Liang ${ }^{1, a}$, Shuang $\mathrm{Wu}^{1, \mathrm{~b}}$, Gui Zhi Sun ${ }^{1, \mathrm{c}}$ \\ ${ }^{1}$ School of Mechanical Engineering, Shenyang University, Shenyang, Liaoning Province, China
}

\begin{abstract}
With the rapid expansion of Chinese container terminal throughput, each container terminal increasingly pays attention to the operational efficiency of storage yard. In this paper, aim at the problem of excessive containers relocation assignments to establish the slotting optimization allocation model, and verify the validity of the model by using Matlab; Using tandem queuing network modeling to build model of the container terminal operating system,and simulate the container terminal logistics system by Witness. It can provide a reference for efficiency improvement of the

container

terminal

operating

system.
\end{abstract}

\section{1 introduction}

The large scale ship of container terminal brings all kinds of pressure to the container terminals. How to solve the problem of the construction and planning of container berths has become the scholars' key focus. Kim and Hong[1] used the heuristic methods and branch and bound method simultaneously to study containers relocation problems. K.H. Kim[2] considered some of the nature of export containers, he mainly used a dynamic programming method to determined the result. Yuewen Gao and Yousan Ji[3] analyzed the weight factor, and used the search technology to show that this method of container terminals had reference value to reduce the number of containers relocation. Jumin Hao[4] builded an optimization model about an inner shell of the container terminal yard, it improved the utilization of terminal yard effectively. Jianfeng Shen[5] proposed palletizing models, including matching rules, most preferred, and the region partition.

The main object of the study is a container terminal logistics system, for containers relocation assignment, mechanical resource configuration issues, a terminal operation system is optimized. Research ideas and methods can provide reference for related wharf optimized operating system.

\section{The study of container terminal containers relocation problem}

\subsection{Model assumptions}

It assumed that the zone and the bay of zone had been allocated in container terminal yard; According to container-delivering information, basic information of all

\footnotetext{
a Corresponding author: author@e-mail.org
}

containers have been identified, including the box weight, port of destination, etc; The containers relocation assignments just were operated during the bay. It assumed that all prepared assigned container are the same type.

\subsection{The establishment and analysis of stockpiling mathematical model}

\subsubsection{Model parameters}

$\mathrm{J}$ expressed the number of stacked inside each Bay; $\mathrm{K}$ represented the number of Bay inside per segment; I expressed the number of stack height inside each Bay. M is represented as a large positive values; $\mathrm{N}$ represented the total number of planned container terminal yard; $d_{n}$ expressed as a destination of the $n$-th container; $w_{n}$ was expressed as an $n$-th grade container carrier; $c_{n}$ expressed as the $n$-th container property value; $v_{k}$ expressed as $k$-th Bay carrying capacity.

$\mathrm{S}_{\mathrm{nkj}}$ noted that the i-layer box position of $\mathrm{j}$ of $\mathrm{k}$-th Bay, which can occupied by the $\mathrm{n}$-th container, With 1 said it had been allocated, with 0 expressing others:

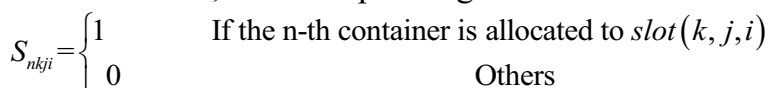
$\mathrm{k}=1,2, \ldots \mathrm{K} ; \mathrm{j}=1,2, \ldots \mathrm{J} ; \mathrm{i}=1,2, \ldots \mathrm{n}=1,2, \ldots$ $\mathrm{N}$.

$R_{k j(i-z)}$ indicated that if there had containers relocation at $\operatorname{slot}(k, j, i)$ and $\operatorname{slot}(k, j, i-z)$ 


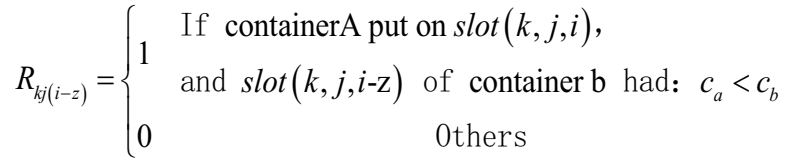

$\mathrm{k}=1,2, \cdots \mathrm{K}, \mathrm{j}=1,2, \cdots \mathrm{J}, \mathrm{i}=2,3, \cdots \mathrm{I}$,

$z=1,2, \cdots i-1$ 。

\subsubsection{Establish the model}

Minimizing the number of containers relocation during the Bay is the goal of container allocation problem.

The objective function:

$$
\mathrm{P}=\min \sum_{\mathrm{J}=1}^{\mathrm{J}} \sum_{i=2}^{I} \sum_{z=1}^{i-1} R_{k j(i-z)}
$$

Restrictions:

$$
\begin{gathered}
\sum_{j=1}^{J} \sum_{i=1}^{I} \sum_{n=1}^{N} S_{n k j i} \leq V_{k} \\
k=1, \quad 2, \ldots \mathrm{K} \text { 。 } \\
\sum_{n=1}^{N} S_{n k j i} \leq \sum_{n=1}^{N} S_{n k j(i-1)} \\
i=2, \quad 3, \ldots \mathrm{I} ; j=1,2, \ldots \mathrm{J} ; \quad k=1,2, \ldots \mathrm{K} 。 \\
\sum_{n=1}^{N} n \cdot S_{n k j i}+\left(1-\sum_{n=1}^{N} S_{n k j i}\right) \cdot M>\sum_{n=1}^{N} n \cdot S_{n k j(i-1)} \\
i=2, \quad 3, \ldots \mathrm{I} ; j=1,2, \ldots \mathrm{J} ; \quad k=1,2, \ldots \mathrm{K} 。
\end{gathered}
$$

Constraints (2) ensured that the container can not be more than the amount of energy they carry large; The constraints (3) expressed that if it is on the second layer container, others should be placed beneath the corresponding container. Constraints (4) ensured that a entering the sequence number of second layer containers was greater than its corresponding approach below the serial number.

This paper used a heuristic to calculate. It was thought as a two-dimensional coordinate system, longitudinal explained to give the container two integer, lengthways explained achieving sequential, the so-called container properties.

The destination A and the weight grade B could be determined site adopted container. Clear the level six weight classes, with a 1-6 six digital representation, $w_{n}=1,2, \ldots, 6$ 。 In a route in accordance with the order of the ports of call were numbered, $d_{n}=1$, $2, \ldots, 7$ 。 If the level was set by weight $w_{n} \quad\left(w_{n}=1\right.$, $2, \ldots, 6), \mathrm{n}$ is the destination $d_{n}\left(d_{n}=1,2, \ldots\right.$, 7 ) , so explained that. $c_{n=}\left(d_{n}, w_{n}, x_{n}, y_{n}\right) \cdot y_{n}$ represented container $n$ ordinate in a cartesian coordinate system, indicating the size of the container transversely value $\mathrm{n}$ in the cartesian coordinate system. The last generation of stacking diagram must simultaneously satisfied the formula (5) and (6).

$$
\left\{\begin{array}{l}
x_{n}<x_{n+l} \\
y_{n}=y_{n+l} \quad \text { 时, } d_{n} \geq d_{(n+l)}
\end{array}\right.
$$

$$
\left\{\begin{array}{l}
x_{n}=x_{n+l} \\
y_{n}<y_{n+l}
\end{array} \text { 时, } w_{n} \leq w_{n+l}\right.
$$

$l=1,2, \cdots-1$ 。

A terminal yard, stacking number $\mathrm{J}=6$, the number of stack $\mathrm{I}=5, V_{K}=30$

Specific steps of the algorithm is: First, choose the number of columns in the order of the first data, the data in the coordinate system of the lower-left corner, select the second point on the top right of the first point. Then the new endpoint of this line as a starting point to repeat the above operation until the condition was not satisfied so far, this would give poly-line one. Secondly, from the remaining points, select the leftmost point between repeated operation of this step, poly-line could be calculated; Then, repeat the above measures, to calculate a set of poly-lines, allowing all the points were arranged in a line of group.

Taking a route Case sequentially port of call as follows: a port - Qingshui - Pusan - Lianyungang Nagoya - Hong Kong - Yokohama. Admission container had six grades weight. Supposing a sequence of random arrival of containers was $\{13,25,16,42,34,32$, $54,64,23,42,51,26,35,51,14,63,45$, $22,64,52,34,26,34,54,63,65$; Follow the proposed algorithm ,It could be get the poly-line, it was shown in Figure 1:

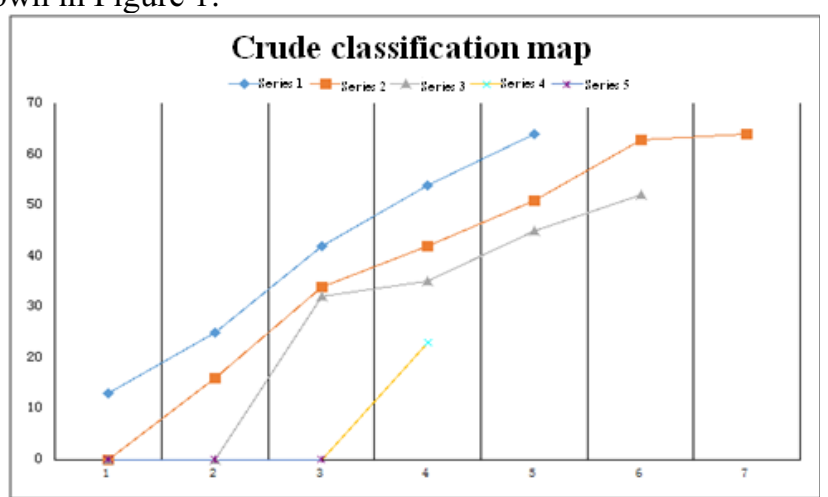

Fig1. Rough classification

According to the poly-line to know the initial state of container, it is shown in Figure 2. As can be seen from this figure, the second and third columns belonged to the long column; the first column, the fourth column and the fifth column were moderate; the sixth column belonged short columns. Over four boxes under ultrahigh column forms obtained were stacked in order with short column by column, the first step was amended, and formed Figure 3: 


\begin{tabular}{l|l|l|l|l|l}
\hline & $65(26)$ & & & & \\
\hline & $64(19)$ & & & & \\
\hline & $63(16)$ & $63(25)$ & & & \\
\hline $64(8)$ & $51(14)$ & $54(24)$ & & & \\
\hline $54(7)$ & $51(11)$ & $52(20)$ & $34(23)$ & & \\
\hline $42(4)$ & $42(10)$ & $45(17)$ & $34(21)$ & $26(22)$ & \\
\hline $25(2)$ & $34(5)$ & $35(13)$ & $26(12)$ & $22(18)$ & \\
\hline $13(1)$ & $16(3)$ & $32(6)$ & $23(9)$ & $14(15)$ & No \\
\hline
\end{tabular}

Fig2. Rough classification

\begin{tabular}{l|l|l|l|l|l}
\hline & & $63(25)$ & & & \\
\hline $64(8)$ & $65(26)$ & $54(24)$ & $34(23)$ & $26(22)$ & \\
\hline $54(7)$ & $64(19)$ & $52(20)$ & $34(21)$ & $22(18)$ & \\
\hline $42(4)$ & $63(16)$ & $45(17)$ & $26(12)$ & $14(15)$ & \\
\hline $25(2)$ & $51(14)$ & $35(13)$ & $23(9)$ & $42(10)$ & \\
\hline $13(1)$ & $51(11)$ & $32(6)$ & $16(3)$ & $34(5)$ & No \\
\hline
\end{tabular}

Fig3. The first correction

According to the above method, the Pareto chart was corrected once again, the results was shown in Figure 4.

\begin{tabular}{l|l|l|l|l|l}
\hline $64(8)$ & $65(26)$ & $63(25)$ & $34(23)$ & $26(22)$ & \\
\hline $54(7)$ & $64(19)$ & $54(24)$ & $34(21)$ & $22(18)$ & \\
\hline $42(4)$ & $63(16)$ & $52(20)$ & $26(12)$ & $14(15)$ & \\
\hline $25(2)$ & $51(14)$ & $45(17)$ & $23(9)$ & $42(10)$ & \\
\hline $13(1)$ & $51(11)$ & $35(13)$ & $16(3)$ & $34(5)$ & $32(6)$ \\
\hline
\end{tabular}

Fig4. The last correction

\section{Container terminal queuing network systems analysis}

\subsection{Determine the container terminal tandem queuing network model}

The relationship between several logistics nodes configuration of the container terminal was shown in Figure 5:

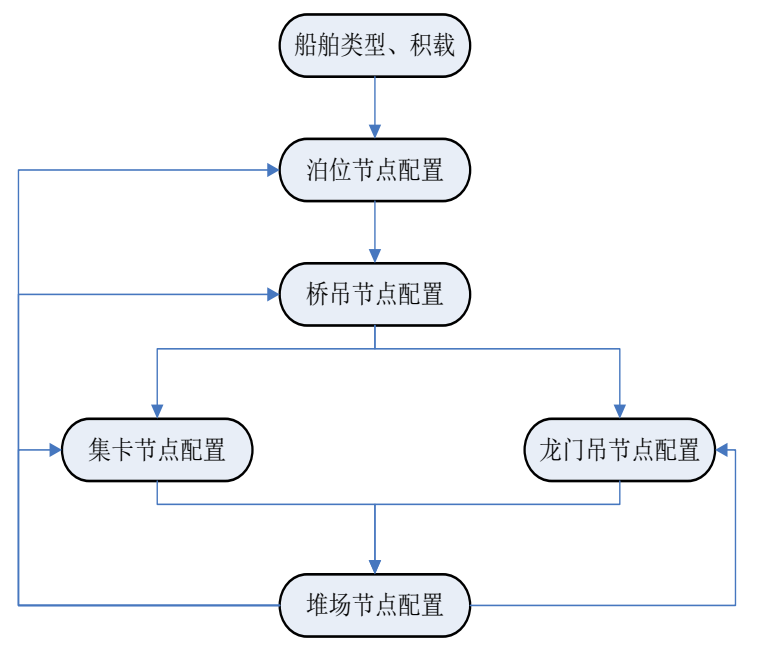

Fig5. Node configure of container terminals system

\subsection{Container Terminal series line network multi- node configuration analysis}

The job level of container terminal logistics network was made up of a plurality of unipolar queue subsystem. Container flows between each node formed a queuing network. According to production of operating line, a series queuing network analytic model was established. Container series line network structure of operating line production was shown in Figure 6 and Figure 7.

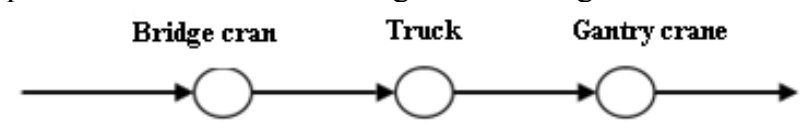

Fig6. Import container terminals system of tandem open queuing network

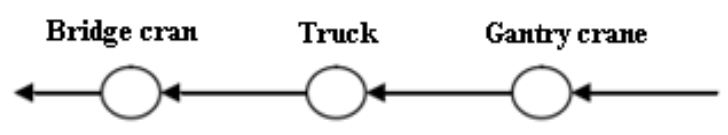

Fig7. Export Container Terminals System of Tandem Open Queuing Network

\section{Container terminal modeling and simulation based on witness}

\subsection{Establish the model}

In this paper, simulate the existing container terminal processes. The main application was the open-loop series multistage queuing network. In the process of import container loading and unloading, First Container loaded onto the truck by crane, Transport the containers shipped under the gantry by the truck, Then place container onto the yard prearranged location by gantry crane. In this paper, the based on container terminal network queuing structure system, Create a system simulation model, and use witness system make the practical operation, summary statistical indicators.

\subsection{Set basic parameters}


The cranes node was the import containers beginning serving node, the gantry crane node was the ending service node, while exports container was opposite. Network can be considered as only one type of customer (container). It was shown in Figure 8:

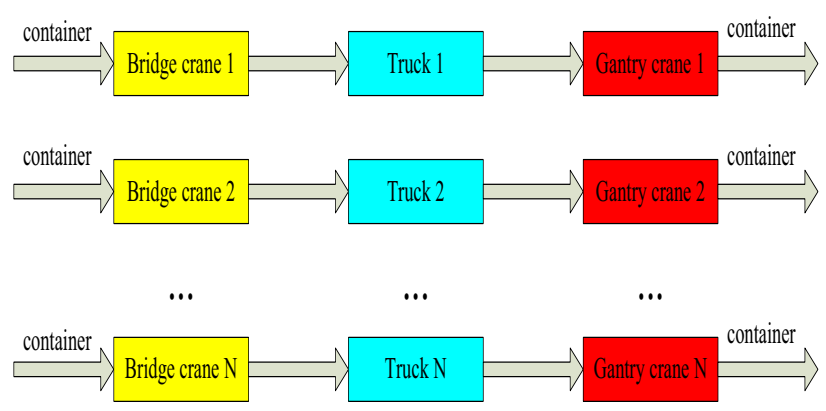

Fig8. Container Terminals System of Tandem Open Queuing Network

The truck arrived the quay crane service center, quay crane was idle, but it might not be able to receive services directly. It was carried out equipping in accordance with the relevant information received in advance of the container ship dock. If one of trucks was matching, the truck was responsible for the work of this operating line .

If the job line was idle, the containers could be set loaded onto the truck. If there were other trucks to went on the loading operation, the truck was set to enter the system waits.

\subsection{The simulation process of witness}

During operation of the model system simulation, container automatically entered the system, when it arrived the bridge node, which obeyed Poisson distribution, it was shown in Figure 9. 1.7 represented that a boat was removed every 1.7 minutes. The containers were transported to the yar to stacking by using the truck. In the simulation, the truck obeyed second-Ireland distribution $(2.0,2,2)$. The simulation time was set at 2 months (60 days).

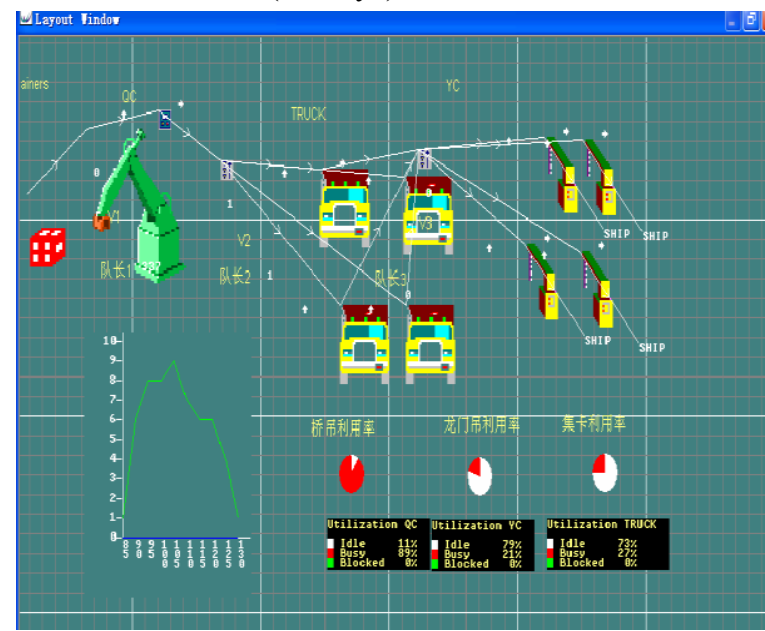

Fig9. Simulation model of mechanism configuration of container terminal

In this paper, choose four ratio to analyze the various indicators, as shown in Table 1, and then from the the average wait time and utilization of equipment to evaluate. Select the most appropriate configuration to improve the efficiency of container terminals.

Table1. Number of Container Terminals Mechanism Configuration

\begin{tabular}{|l|l|l|l|l|}
\hline $\begin{array}{l}\text { Crane: Truck: } \\
\text { gantry crane }\end{array}$ & $(1: 4: 4)$ & $(1: 5: 5)$ & $(1: 5: 4)$ & $(1: 4: 5)$ \\
\hline Crane utilization & $85 \%$ & $85 \%$ & $83 \%$ & $84 \%$ \\
\hline Truck utilization & $25 \%$ & $20 \%$ & $20 \%$ & $25 \%$ \\
\hline $\begin{array}{l}\text { Gantry crane } \\
\text { utilization }\end{array}$ & $19 \%$ & $15 \%$ & $19 \%$ & $15 \%$ \\
\hline Buffers01 & 211455 & 202103 & 215442 & 206365 \\
\hline Buffers02 & 77 & 4 & 4 & 87 \\
\hline Buffers03 & 28 & 2 & 42 & 0 \\
\hline
\end{tabular}

\subsection{Analysis of test results}

It could be observed from simulation, when different ratios, through objective analysis option 1: 5: 5 was the best configuration, the equipment utilization was higher, queue length was relatively short.

\section{Conclusion}

First, the optimization stockpiling mathematical model was established. Using MATLAB to select a randomly data, Use optimized model to stacking containers, Simulate the process of shipment suitcase, and get the most optimal stockpiling mathematical model.Second, the paper created a container terminal simulation model based on series line network. Based on the historical operating data of a container terminal, the model was established according to the reasonable options of this paper, and use WITNESS to make simulation experiments. The test effectively demonstrated the effectiveness of the proposed methods, there had a good reference for the scheduling of container handling equipment.

\section{References}

[1] Zhao Dan.Review and Analysis on Institutional Management for Global Ports[J]. JOURNAL OFNINGBO UNIVERSITY,2006.3(2):36-46.

[2] E.mcdowilll,gmartind.cho,west. A study of maritime container handing Oregon state university. [J]Sea grant college program ads Coregon.2011.2(2):3-6.

[3]Peter Preston, Erhan Kozan. An approach to determine storage locations of containers at seaport terminals[J]. Computers\&Operations Research. 2013,27(1):13-32.

[4]Ying Xie. Port container yard stacking optimization model and algorithm[J].Journal of Central South University,2011 30(2): 78-119.

[5]Jumin Hao, Zhuoshang Ji, Yan lin. The order of mixing job Yard BAY Optimization Model[J]. Advancesin Systems Science and Applications,2000, 40(1): 102-105. 